\title{
Measurement of Linewidth Enhancement Factor of Semiconductor Lasers Using an Injection-Locking Technique
}

\author{
G. Liu, X. Jin, and S. L. Chuang, Fellow, IEEE
}

\begin{abstract}
A new method for measuring the linewidth enhancement factor of semiconductor lasers using the injection-locking technique is presented. This idea is based on the relation between the upper and lower bounds of the locked and unlocked regimes when the detuning of the pump and slave laser is plotted as a function of the injection power. Our results are confirmed with an independent measurement using amplified spontaneous emission (ASE) spectroscopy as well as our theory, which takes account of the realistic quantum-well $(\mathrm{QW})$ band structure and many-body effects. This method provides a new approach to measure the linewidth enhancement factor above laser threshold.
\end{abstract}

Index Terms-Linewidth enhancement factor, optical injection, semiconductor laser.

$\mathbf{T}$ HE LINEWIDTH enhancement factor $(\alpha)$ is one of the key parameters for semiconductor lasers. The linewidth enhancement factor characterizes the linewidth broadening and chirp due to fluctuation in the carrier density, which are detrimental sources for high-speed performance. Several methods have been proposed to measure $\alpha$, such as interferometric measurement [1], RF-modulation measurement [2], injection-locking method [3], and amplified spontaneous emission (ASE) method [4]. In this letter, a new method for the determination of the linewidth enhancement factor of a semiconductor Fabry-Perot (FP) laser is performed by measuring the injection locking range of the laser. In the past, it was proposed that $\alpha$ can be obtained by measuring the optical power change of an injection-locked laser [3]. In this method, the injection level should be low to satisfy the assumption for the $\alpha$ calculation. At the same time, it is difficult to injection-lock the laser and measure the power variation at low injection levels accurately. Our method does not require any fitting parameters, which would bring uncertainty for different laser systems. Meanwhile, our results are in excellent agreement with our independent measurement based on ASE spectroscopy. Both experimental results are confirmed with our theory, which takes account of realistic band structure with the valence band-mixing effect and many-body effects.

In an optical injection-locked laser system, which consists of a master laser (stable laser oscillation) and a test laser, the locking properties of the test laser depend on its linewidth enhancement factor $\alpha$. The locking range is asymmetric when

Manuscript received December 15, 2000; revised January 25, 2001. The authors are with the Department of Electrical and Computer Engineering, University of Illinois at Urbana-Champaign, Urbana, IL 61801 USA. Publisher Item Identifier S 1041-1135(01)03766-1.

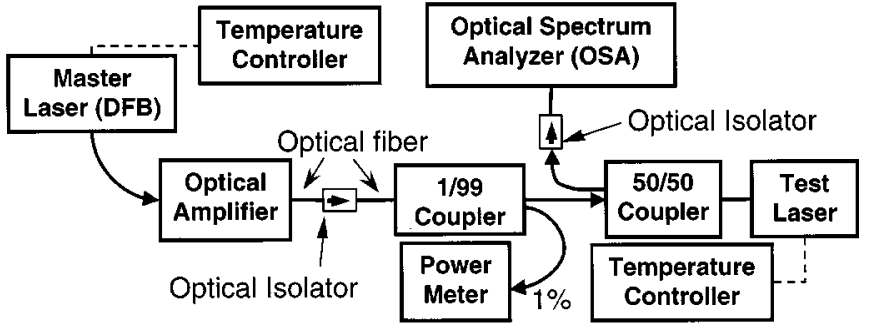

Fig. 1. Experimental setup for measuring the linewidth enhancement factor of semiconductor FP lasers using injection locking technique.

$\alpha \neq 0$, and the value of the linewidth enhancement factor can be evaluated from this asymmetry.

Fig. 1 shows the experimental setup. The test laser is a buried heterostructure FP laser with five $-0.78 \%$ compressively strained quantum wells (QWs) made of $\operatorname{In}_{1-x-y} \mathrm{Ga}_{x} \mathrm{Al}_{y}$ As materials with a lasing wavelength at $1550 \mathrm{~nm}$ [5]. The test laser has a threshold current of $11.7 \mathrm{~mA}$, and is operated at a bias current of $25 \mathrm{~mA}$ and a heat sink temperature of $25^{\circ} \mathrm{C}$. The master lasers are selected from a group of single wavelength DFB lasers with sidemode suppression ratios greater than 40 $\mathrm{dB}$ and lasing wavelengths ranging from $1520 \mathrm{~nm}$ to 1580 $\mathrm{nm}$. The master laser is mounted on a heat sink. The heat sink temperature is used to control the lasing wavelength of the master laser with a tunability of about $1 \mathrm{~nm}$. The light is coupled into an optical fiber by a laser optical-fiber interface (LOFI) and fed into an erbium-doped fiber amplifier (EDFA). In this way, the wavelength and optical power of the injection laser light can be controlled independently. The $1 \%$ output of a $1 \times 2$ fiber coupler $(1 \% / 99 \%)$ is connected to an optical power meter in order to monitor the injected light power. The light then is injected into the test laser. An optical spectrum analyzer (OSA) is used to monitor the spectrum of the test laser.

Fig. 2 shows the optical power spectra of the test laser. The master laser used in this figure lases at $1534.2 \mathrm{~nm}$, which is close to one of the free-running FP modes of the test laser $(1534.35 \mathrm{~nm})$. The injection power in this figure represents the master laser power measured before being coupled into the test laser. The long dashed line shows the free-running laser emission spectrum of the test laser. When the external master laser light is injected into the test laser, which is biased above threshold, the injected light competes with the optical gain of the test laser for amplification. At a given detuning between the external laser light frequency and one of the FP mode frequencies of the test laser, when the optical power of 


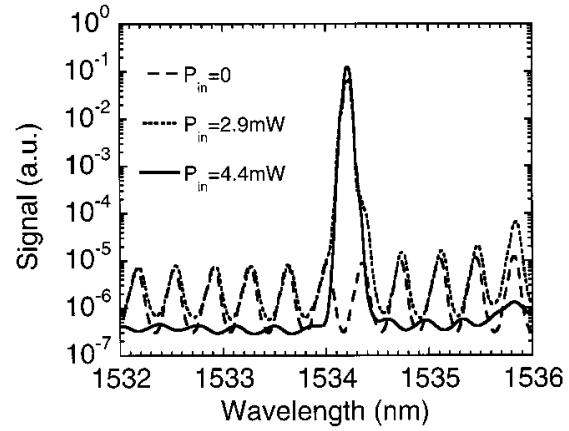

Fig. 2. Optical spectra of the free-running state (long dashed line), the unlocked state (biased at $25 \mathrm{~mA}, 25^{\circ} \mathrm{C}$ under an injection optical power of $2.9 \mathrm{~mW}$ (short dashed line), and the injection-locked state under an injection optical power of $4.4 \mathrm{~mW}$ (solid line) for the test laser.

the injected light is weak, the light at the injection wavelength is barely amplified. When the injected optical power is strong enough and close to the FP mode of the test laser (1534.35 $\mathrm{nm})$, the injection-locked state of the test laser is achieved. The injection-locked state is determined by the observations that the test laser lases at the injection laser wavelength and all the other free-running sidemodes are greatly suppressed. Once an injection-locking state is reached, almost all of the power of the test laser is emitted at the optical frequency of the master laser, as shown by the solid line in Fig. 2. In contrast to this, when the injected optical power is not strong enough to lock the test laser, the spectrum of the unlocked test laser injection (short dashed line in Fig. 2) shows that the optical spectrum of the test laser and the master laser are approximately added together. The frequencies of the cavity mode of the test laser shift very little by the external signal. The conditions which allow the injection-locking occur will enable us to measure the linewidth enhancement factor for the test laser accurately.

The overall locking range of a semiconductor laser is determined by both detuning $\left(\Delta \omega=\omega_{i}-\omega_{0}\right)$ and the external injection power. From the rate equations, an asymmetric locking bandwidth can be obtained [6]

$$
\begin{aligned}
\Delta \omega_{\min } & =-\frac{c}{2 n_{\mathrm{e}} L} \sqrt{\frac{S_{\mathrm{i}}}{S}\left(1+\alpha^{2}\right)}<\Delta \omega<\frac{c}{2 n_{\mathrm{e}} L} \sqrt{\frac{S_{\mathrm{i}}}{S}} \\
& =\Delta \omega_{\max }
\end{aligned}
$$

where

$$
\begin{array}{ll}
S_{\mathrm{i}} & \text { injected optical power; } \\
S & \text { test laser optical power; } \\
n_{\mathrm{e}} & \text { refractive index; } \\
L & \text { laser cavity length; } \\
c & \text { speed of light. }
\end{array}
$$

Outside this region is the unlocked region, where the injection level is too low or the detuning is too high to reach injection-locking condition. A detailed stability analysis can be found in [7], which also calculates a self-pulsation zone (a Hopf bifurcation), chaotic zone, and a coherence collapse zone. In this letter, we focus on the stable locking regime. As a note, our experiment is carefully controlled to make sure that the injected power is not strong enough to cause self pulsation. Also, the effect due to the nonlinear gain suppression is controlled

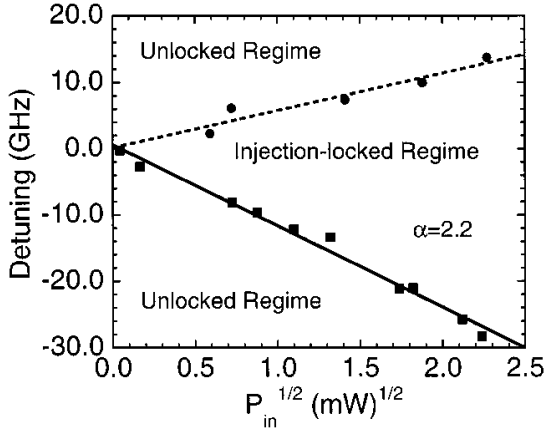

Fig. 3. The upper and lower detuning ranges are plotted as functions of the square root of the injected optical power for the boundaries between the locked and unlocked states. The ratio of the two slopes, which is independent of the optical powers, is used to calculate the linewidth enhancement factor.

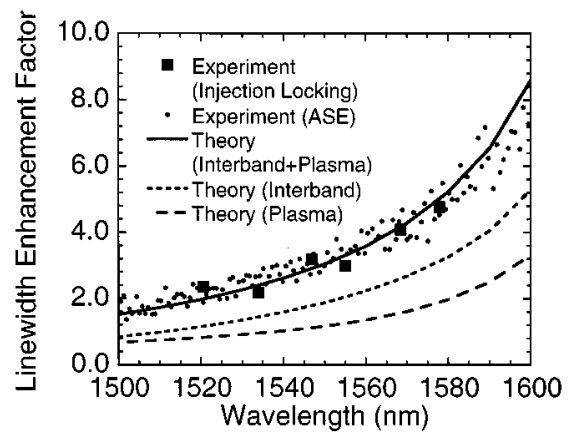

Fig. 4. The linewidth enhancement factor is plotted as a function of the wavelength for the test laser. The solid squares are the experimental results using the injection locking technique, the dots are the experimental data using the ASE spectroscopy. The theoretical linewidth enhancement factor is plotted for the interband contribution (short dashed line), the plasma effect contribution (long dashed line), and their total (solid line).

to be less than $3 \%\left(1+\epsilon S+\epsilon S_{\mathrm{i}}<1.03\right)$. The incomplete locked regime (as discussed in [6]), in which the energy is distributed in the free-running and locked modes, is considered the unlocked regime so that (1) can be applied. From (1), as shown in Fig. 3, the upper and lower detuning ranges are plotted for the conditions that the injection-locking occurs at the wavelength of $1534.35 \mathrm{~nm}$ (one of the free-running FP modes of the test laser). The horizontal axis is the square root of the injected optical power. From the ratio of the two slopes for the boundaries of the locking ranges (least square fit), which is independent of the optical powers, the linewidth enhancement factor of the test laser can be found to be $\alpha=2.2$. Such experiments are performed over wavelengths ranging from 1520 to $1580 \mathrm{~nm}$ with different DFB lasers as the master laser. The linewidth enhancement factor is plotted in square symbols in Fig. 4.

In order to verify the above results, we also perform measurement of the linewidth enhancement factor using the ASE spectroscopy. The ASE spectrum of the test laser is measured for two close currents below lasing threshold at $T=25^{\circ} \mathrm{C}$. The peak wavelength of each FP mode in the ASE spectrum is a function of the effective refractive index $n_{\mathrm{e}}(x)$. The change in the effective index can be calculated from [8]

$$
\Delta n_{\mathrm{e}}=\frac{\lambda}{2 L} \frac{\Delta \lambda_{l}}{\Delta \lambda_{m}}
$$


where $\Delta \lambda_{l}$ is the wavelength shift of an FP mode due to a change in injected carriers at two bias currents. $\Delta \lambda_{m}$ is the mode spacing of two adjacent FP peaks. $\alpha$ can then be extracted using

$$
\alpha=-\frac{4 \pi}{\lambda} \frac{\Delta n_{\mathrm{e}}}{\Delta G_{\text {net }}}
$$

where $\Delta G_{\text {net }}$ is change of the net modal gain [5]. The results are plotted as dots in Fig. 4, and they are in excellent agreement with our results using the injection-locking technique. When using the ASE spectroscopy method, the linewidth enhancement factor is measured near threshold condition. On the other hand, using the injection locking method, the linewidth enhancement factor is measured above threshold and lasing condition. For high-speed modulation applications, the linewidth enhancement factor measured at the lasing condition will be more important. The results show that the linewidth enhancement factor does not change too much near threshold and at lasing conditions.

To model the $\mathrm{In}_{1-x-y} \mathrm{Ga}_{x} \mathrm{Al}_{y}$ As QW lasers, the band structures of the quantum wells are calculated using a block-diagonalized $3 \times 3$ Hamiltonian based on the $\mathbf{k} \cdot \mathbf{p}$ method for valence subbands and a simple isotropic parabolic band model for conduction subbands. Once the band structure is known, the optical gain $g(\omega)$ based on a non-Markovian gain model using a spontaneous-emission transformation method is given in[5], and the induced change in the refractive index $\delta n_{\mathrm{e}}^{\mathrm{int}}(\omega)$ due to interband transition is given by

$$
\begin{aligned}
\delta n_{\mathrm{e}}^{\mathrm{int}}(\omega)= & \sqrt{\frac{\mu_{o}}{\epsilon}}\left(\frac{e^{2} c}{2 m_{o}^{2} \omega^{2}}\right) \\
& \times \sum_{\sigma, l, m} \int_{0}^{\infty} d k_{t} \frac{k_{t}}{\pi L_{z}}\left|M_{l m}^{\sigma}\right|^{2}\left(f_{l}^{c}\left(k_{t}\right)-f_{m}^{\sigma}\left(k_{t}\right)\right) \\
& \times\left[-\frac{\left(1-\operatorname{Re} q_{k_{t}}\right) \operatorname{Im} L+\operatorname{Im} q_{k_{t}} \operatorname{Re} L}{\left(1-\operatorname{Re} q_{k_{t}}\right)^{2}+\left(\operatorname{Im} q_{k_{t}}\right)^{2}}\right]
\end{aligned}
$$

where definitions of symbols can be found in [5]. In addition, there is a contribution from the free carrier plasma effect [9] for TE polarization.

$$
\delta n_{\mathrm{e}}^{\text {plasma }}(\omega)=-\frac{e^{2} N}{2 m_{\mathrm{r}}^{*} \omega^{2} n_{\mathrm{r}} \epsilon_{0}}
$$

where

$N \quad$ carrier concentration;

$n_{\mathrm{r}} \quad$ refractive index of the QW layer;

$m_{\mathrm{r}}^{*} \quad$ reduced effective mass for electrons and holes.

The linewidth enhancement factor due to the interband transition is then obtained by

$$
\alpha=-\frac{4 \pi}{\lambda} \frac{\partial \delta n_{\mathrm{e}} / \partial N}{\partial g / \partial N}
$$

where the total refractive index change is $\delta n_{\mathrm{e}}=$ $\delta n_{\mathrm{e}}^{\mathrm{int}}+\delta n_{\mathrm{e}}^{\text {plasma }}$ for the TE mode, which is the domi- nant optical mode for the compressively strained QW laser as verified by the experiment.

We have fitted the optical gain for this laser in [5]. Using the same fitting parameters, the change of the refractive index change due to the interband transition is calculated. In Fig. 4, the linewidth enhancement factor due to the interband transition (short-dashed line), the plasma effect (long-dashed line), and their sum are plotted. The theoretical curve (interband plus plasma effects) agree with the experiment very well. Both the interband transition and the plasma effect contribute to the total linewidth enhancement factor significantly for the laser studied. At the laser wavelength of $1550 \mathrm{~nm}$, the linewidth enhancement factor is 3.0. This value agrees with typical value of long wavelength strained QW lasers. The experimental uncertainty from both experiments is about $15 \%$.

The injection-locking technique itself has a great potential for increasing the laser modulation bandwidth, decreasing the chirp, and measuring many fundamental parameters of semiconductor lasers [10], [11]. Here, we explored the application of this technique for measuring $\alpha$ of a long-wavelength $\mathrm{In}_{1-x-y} \mathrm{Ga}_{x} \mathrm{Al}_{y}$ As strained QW laser. Without the absolute values of the injection level, the value of $\alpha$ can be evaluated from the detuning ranges of the injection locking. Our theory shows good agreement with experimental results.

\section{REFERENCES}

[1] J. Ehrhardt, A. Villeneuve, G. I. Stegeman, H. Nakajima, J. Landreau, and A. Ougazzaden, "Interferometric measurement of the linewidth enhancement factor of a $1.55 \mu \mathrm{m}$ strained multi-quantum-well InGaAs/InGaAsP amplifier," IEEE Photon. Technol. Lett., vol. 4, pp. 1335-1338, Dec. 1992.

[2] H. Li, "RF-modulation measurement of linewidth enhancement factor and nonlinear gain of vertical-cavity surface-emitting lasers," IEEE Photon. Technol. Lett., vol. 8, pp. 1594-1596, Dec. 1996.

[3] K. Iiyama, K. Hayashi, and Y. Ida, "Simple method for measuring the linewidth enhancement factor of semiconductor lasers by optical injection locking," Opt. Lett., vol. 17, pp. 1128-1130, Aug. 1992.

[4] T. C. Newell, D. J. Bossert, A. Stintz, B. Fuch, K. J. Malloy, and L. F. Lester, "Gain and linewidth enhancement factor in InAs Quantum-dot laser diodes," IEEE Photon. Technol. Lett., vol. 11, pp. 1527-1529, Dec. 1999.

[5] J. Minch, S. H. Park, T. Keating, and S. L. Chuang, "Theory and Experiment of $\operatorname{In}_{1-x-y} \mathrm{Ga}_{x} \mathrm{As}_{y} \mathrm{P}_{1-y}$ and $\mathrm{In}_{1-x-y} \mathrm{Ga}_{x} \mathrm{Al}_{y}$ As long-wavelength strained quantum-well lasers," IEEE J. Quantum Electron., vol. 35, pp. 771-782, May 1999.

[6] I. Petitbon, P. Gallion, G. Debarge, and C. Chabran, "Locking bandwidth and relaxation oscillations of an injection-locked semiconductor laser," IEEE J. Quantum Electron., vol. 24, pp. 148-154, Feb. 1988.

[7] V. Annovazzi-lodi, A. Scire, M. Sorel, and S. Donati, "Dynamic behavior and locking of a semiconductor laser subjected to external injection," IEEE J. Quantum Electron., vol. 34, pp. 2350-2357, Dec. 1998.

[8] C. S. Chang, S. L. Chuang, J. R. Minch, W. W. Fang, and Y. K. Chen, "Amplified spontaneous emission spectroscopy in strained quantum-well lasers," IEEE J. Select. Topics Quantum Electron., vol. 1, pp. 1100-1107, Dec. 1995.

[9] Y. Huang, S. Arai, and K. Komori, "Theoretical linewidth enhancement factor alpha of GaInAs/GaInAsP/InP strained-quantum-well structures," IEEE Photon. Technol. Lett., vol. 5, pp. 142-145, Feb. 1993.

[10] X. Jin and S. L. Chuang, "Enhancement of modulation bandwidth in semiconductor quantum-well lasers by injection locking," IEEE $J$. Quantum Electron., submitted for publication.

[11] J. M. Liu and T. B. Simpson, "Characterization of fundamental parameters of a semiconductor laser with an injected optical probe," IEEE Photon. Technol. Lett., vol. 5, pp. 380-382, Apr. 1993. 